\title{
VARIATION OF CONTACT ANGLE AND STRUCTURE WITH MOLECULAR LENGTH AND SURFACE DENSITY IN ADSORBED FILMS OF FATTY ACIDS
}

\author{
W. C. Bigelow and L. O. Brockway \\ Department of Chemistry, University of Michigan, Ann Arbor, Michigan
}

Received November 29,1955

\begin{abstract}
Films of fatty acids from 12 to 22 carbons were prepared by adsorption on glass microscope slides from cetane solutions. Variations in density were achieved (1) by using a range of concentrations with a fixed short time of immersion and (2) by subsequent immersion in cetane of the most dense films.

Electron diffraction patterns showed an increasing average tilt of the molecules relative to the surface normal with decreasing length of the molecules but no apparent change in tilt with variations in surface density. The contact angles exhibited by drops of cetane on the films decreased markedly from a maximum of $43-44^{\circ}$ when the density of the adsorbed molecules on the surface was decreased, but were independent of the variations in tilt and length of the molecules.
\end{abstract}

\section{INTRODUCTION}

Previous investigations $(1,2)$ have shown that polar organic compounds, such as the fatty acids and the corresponding alcohols, amines, and amides, adsorb from solution in nonpolar organic solvents onto clean solid surfaces to form films which have the interesting property of being very poorly wetted by the solutions and the pure nonpolar solvents. As the film-covered surfaces are withdrawn from the solutions, the liquid recedes, leaving the film-covered surfaces dry. Drops of the solutions or of the pure nonpolar solvents which are subsequently placed on the surfaces show contact angles of as much as $40^{\circ}$ and move about freely as the surfaces are tilted. To describe these properties briefly the films are commonly referred to as being "oleophobic."

Although these oleophobic films have been studied rather extensively, their detailed structure has not yet been determined nor related to their oleophobic properties. In the early studies of the formation and properties of the films (1), it was concluded that they consisted of monolayers of closely packed polar molecules in which the molecules were attached to the surfaces by their polar groups and were oriented with their hydrocarbon chains perpendicular to the substrate surfaces. The outer surfaces of the 
films were thus considered to consist of a nearly continuous array of methyl groups, and the difference in the character of this methyl-rich surface and the predominantly methylenic organic liquids was proposed as the basic reason for the incomplete wetting of the hydrocarbon films by the nonpolar hydrocarbon liquids. Subsequent electron diffraction studies $(3,4)$ confirmed these basic structural concepts, but showed that the molecules were, on the average, tilted several degrees in random directions from the surface normal, and evidence was obtained indicating that the average tilt varied depending on the roughness of the substrate surfaces. More recently (5) electron microscope studies of the films have led to the proposal of a structural model in which the molecules associate in cluster or micelles about 100 A. in diameter. Owing to the difference in the diameters of the hydrocarbon chains and the polar groups, this model proposes that the molecules tilt toward the centers of the micelles somewhat like the corn stalks in a corn shock. This general arrangement for the molecules is consistent with observations on the rate of evaporation of the adsorbed molecules from the surfaces at elevated temperatures (6). A further interesting feature of this model is the proposal that the average tilt of the adsorbed molecules should decrease as the length of the hydrocarbon chains of the molecules increases.

The present investigation was undertaken to obtain more information on the factors affecting the structures and the oleophobic properties of the films. The principal factors which have been considered here are: $(1)$ variations in the length of the polar molecules and (2) variations in the average density of the polar molecules on the surfaces. Studies have been made of films of each of the fatty acids from 12 to 22 carbon atoms in length. In addition the films have been prepared and treated in ways designed to introduce systematic variations in the average density of molecules on the surfaces. The structures of the films were examined by electron diffraction, and their oleophobic properties evaluated by measuring the advancing contact angles of drops of cetane placed on the films.

\section{Experimental Methods}

The films studied here were prepared on glass microscope slides by adsorption from cetane solutions. The fatty acids used include: lauric, myristic, palmitic, stearic, arachidic, and behenic acids. These acids were furnished by the Chemical Division of the Procter and Gamble Company and were all reported to be at least $95 \%$ pure. Their respective melting points were: $44.1^{\circ}, 57.5^{\circ}, 63.7^{\circ}, 69.4^{\circ}, 76.0^{\circ}$, and $80.5^{\circ} \mathrm{C}$. The cetane was a commercial grade material having a melting range of $17.3^{\circ}-17.8^{\circ} \mathrm{C}$. To insure its freedom from adsorbable impurities, the cetane was passed repeatedly through columns of activated silica gel and alumina until drops placed on both strongly alkaline and strongly acid water showed no tendency to spread. This has been shown to be a satisfactory test for the ab- 
sence of polar impurities for solvents used in preparing films by adsorption from solution (1).

Glass microscope slides were chosen as substrates for the films because electron microscopic studies (7) have shown their surfaces to be highly polished to a uniformly smooth condition, and it was hoped to avoid variations in the roughness of the substrate and to eliminate the tedious polishing procedures which would be necessary to produce comparable surfaces on metals or minerals.

Some difficulty was encountered in cleaning the slides so that they would adsorb films having reproducible properties. After considerable experimentation, a procedure was adopted consisting of the following sequence of operations: (1) thorough washing in a hot detergent (Tide) solution, (2) thorough rinsing with hot water, (B) immersion for 10 to 15 minutes in cold chromic-sulfuric acid cleaning solution, (4) thorough rinsing with steaming distilled water running directly from a still equipped with a block-tin condenser, (5) immersion for 30 minutes in hot distilled water followed by a final rinse with the steaming distilled water. The slides were then hung at one corner with stainless steel wire clips in a clean drying oven with a stainless steel interior and were dried at $150^{\circ} \mathrm{C}$. for 4 hours. Upon removal from the oven they were stored until use in glass-stoppered bottles. In all these operations the slides were handled with acid-cleaned, glass-tipped tweezers, and extreme care was exercised to avoid scratching or marring their surfaces. Even with most careful adherence to this procedure variations were occasionally observed in the behavior of slides cleaned in different batches. Therefore, slides for a given series of experiments were cleaned at the same time, and comparisons have been made only for slides cleaned in the same batch. All the glass-stoppered bottles and other glassware used in these experiments were cleaned by a process similar to that used with the slides.

The electron diffraction examinations were made by the reflection method using a General Electric electron diffraction instrument operating at an electron-accelerating potential of $35 \mathrm{kv}$. Some difficulties were encountered owing to the accumulation of electrostatic charges on the nonconducting glass slides. The usual procedure of bombarding the surfaces with lowenergy electrons was found to destroy the monolayers in many instances and therefore could not be used. It was found that good patterns could be obtained by using a low-intensity electron beam and an angle of incidence of the beam relative to the slides which was somewhat larger than is generally used in reflection studies. Consequently, the first-order reflections from the adsorbed molecules were usually masked by the shadow of the slides, and the patterns showed a rather high background which caused some difficulty in the analysis of the patterns. The interpretation of the electron diffraction patterns in terms of the structure of the monolayers has been described by Karle and Brockway (4). 
The contact angles of the cetane drops were determined by the sessile drop, or drop-on-plate, method. To insure that the drops exhibited advancing angles, they were formed on the surfaces with a hypodermic syringe which was operated through micrometer screw controls permitting the tip of the syringe to be slowly withdrawn from the drops while the cetane was being ejected and the drops were growing in size. The contact angles $(\theta)$ of the drops were calculated from their maximum heights $(h)$ and maximum widths $(w)$ using the formula $\tan \theta / 2=2 h / w$. This relationship is valid for drops having the shape of spherical segments but has been shown by Mack (8) to be applicable in general only to drops which are less than 0.5 $\mathrm{mm}$. in diameter. To insure compliance with these conditions, all of the drops measured here were smaller than this. The drop dimensions were measured with a horizontal telescope equipped with a $5 \times$ objective and a $12.5 \times$ filar micrometer eyepiece. With this instrument measurements could be reproduced to $\pm .002 \mathrm{~mm}$., which corresponds to an uncertainty of no more than $0.5^{\circ}$ in the determination of the contact angles.

\section{Results}

Two series of experiments were used to study the effects of the average density of the adsorbed molecules on the structure and oleophobic properties of the films. The first of these, which also included studies of the effects of variations of the length of the adsorbed polar molecules, was carried out with films of each of the fatty acids prepared from solutions ranging in concentrations from $1 \times 10^{-4}$ to $5 \times 10^{-2}$ molal, using a constant period of immersion of 5 seconds for depositing the films from each solution. The period of immersion was chosen relatively short so that it would be unlikely that the adsorption process would be completed in the more dilute solutions. The density of adsorbed molecules would then increase with increasing concentration of the solutions.

TABLE I

Average Advancing Contact Angles for Drops of Cetane on Fatty Acid Films Deposited on Glass Surfaces by 5 -Second Immersion in Solutions of Different Concentration ${ }^{a}$

\begin{tabular}{l|c|c|c|c|c|c}
\hline \multirow{2}{*}{ Acid } & \multicolumn{6}{|c}{ Molal concentrations of solutions } \\
\cline { 2 - 7 } & $1 \times 10^{-4}$ & $5 \times 10^{-4}$ & $1 \times 10^{-3}$ & $5 \times 10^{-3}$ & $1 \times 10^{-2}$ & $5 \times 10^{-2}$ \\
\hline Behenic & - & - & $44^{\circ}$ & N.S. & N.S. & N.S. \\
Arachidic & $43^{\circ}$ & $43^{\circ}$ & $44^{\circ}$ & N.S. & N.S. & N.S. \\
Stearic & $42^{\circ}$ & $44^{\circ}$ & $44^{\circ}$ & $44^{\circ}$ & N.S. & N.S. \\
Palmitic & N.O. & N.O. & $41^{\circ}$ & $44^{\circ}$ & $44^{\circ}$ & N.S. \\
Myristic & N.O. & N.O. & $36^{\circ}$ & $43^{\circ}$ & $44^{\circ}$ & N.S. \\
Lauric & N.O. & N.O. & $25^{\circ}-36^{\circ}$ & $39^{\circ}$ & $42^{\circ}$ & $43^{\circ}$ \\
\hline
\end{tabular}

${ }^{a}$ Each value is the average for not less than 5 different drops on each of 5 different slides; the individual measurements lay within $\pm 1^{\circ}$ of the average (except for lauric acid at $10^{-8}$ molal).

N.O.-not oleophobic.

N.S.-indicated concentration exceeds solubility. 
The results of the contact angle measurements on the films formed under these conditions are summarized in Table I. Films prepared from sufficiently concentrated solutions of each acid showed maximum contact angles of $43-44^{\circ}$. The concentrations required for this were near the solubility limits for the various acids at room temperature and ranged from about $1 \times 10^{-3}$ molal for arachidic acid to about $5 \times 10^{-2}$ molal for lauric acid. With decreasing solution concentrations, the contact angles decreased, the effect being somewhat greater for the shorter acids than for the longer ones. Below concentrations ranging from about $1 \times 10^{-3}$ molal for lauric and myristic acids to about $1 \times 10^{-4}$ molal for stearic and arachidic acids, periods of immersion greater than 5 seconds were required to produce oleophobic films.

Electron diffraction studies were made on each of the slides after completion of the contact angle measurements and on several additional slides prepared immediately before insertion into the diffraction camera. Diffraction patterns consisting of well-defined, arced layer lines were obtained from all films except those of lauric acid. These patterns are similar to those reported by Brockway and Karle (3) in previous studies of acid films deposited by adsorption from solution, and by Germer and Storks (9) in studies of monolayers deposited by the Langmuir-Blodgett technique. For reasons as yet unknown, the photographs from the slides bearing the lauric acid films contained no features characteristic of long-chain organic molecules. The diffraction patterns from the other acids were analyzed, using the general principles outlined by Karle and Brockway (4), for indications of variations in the arrangement of the adsorbed molecules. Comparison of patterns from monolayers of the different fatty acids showed a slight increase in the curvature of the layer lines as the length of the fatty acids decreased. This is indicative of an increase in the average tilt of the molecules from the surface normal as the length of the hydrocarbon chains decreases. The average tilts estimated for the different acids are as follows: behenic and arachidic, $2^{\circ}$; stearic, $4^{\circ}$; palmitic, $6^{\circ}$; myristic, $8^{\circ}$. These values were arrived at by estimating the maximum slope of the layer lines in a number of patterns for each acid, averaging, and considering this angle to be approximately equal to the average molecular tilt of the molecules. The general trend indicating an increase in the average tilt with a decrease in chain length of the molecules is considered to be reliable, although the separate values for the angles are probably not better than $\pm 3^{\circ}$. It is probable that the maximum molecular tilt in each case is as much as twice these average values. There were no detectable differences in the arcing of the layer lines in the patterns from solutions of different concentrations of the same acid, though the layer lines in patterns from the films prepared from the more dilute solutions were generally less intense relative to the background scattering than those from films prepared from the more concentrated solutions. 
Slides which were immersed in the very dilute acid solutions for periods too short to produce oleophobic films were also examined by electron diffraction for evidence of oriented adsorbed molecules. A $1 \times 10^{-5}$ molal arachidic acid solution failed to produce oleophobic surfaces on slides immersed for a period of 15 minutes. After the liquid remaining on these slides was removed by centrifugation, very weak layer line diffraction patterns were obtained. Slides immersed in this solution for periods of 30 minutes and 3 hours were oleophobic over parts of their surfaces and gave somewhat stronger layer line patterns. Slides immersed for 20 hours were oleophobic over their entire surfaces and produced layer line diffraction patterns of intensities comparable to those from films prepared from the more concentrated solutions. All these patterns were carefully compared with those obtained from films prepared from the more concentrated solutions, and there was no noticeable difference in the arcing of the layer lines.

The second series of experiments to determine the effects of variations in the molecular densities of the films on their structure and properties was made with films of arachidic and stearic acids. These films were prepared from the $1 \times 10^{-3}$ molal solutions, using a 5 -second period of immersion, and were subsequently immersed for short periods in pure cetane to redissolve some of the adsorbed molecules. It was found that the films would generally retain their oleophobic properties after immersion in the cetane for periods of as much as 7 to 10 minutes, but that the contact angles decreased from the initial values of $43-44^{\circ}$ to values just above $30^{\circ}$ as the period of immersion was increased within these limits. Contact angle values observed in typical experiments with each acid are given in Table II. Each of these values is the average for measurements made on five or more drops. Electron diffraction patterns obtained from the depleted films were care-

TABLE II

Advancing Contact Angles ( $\theta$ ) of Drops of Cetane on Fatty Acid. Films Before and After Immersion in Cetane

\begin{tabular}{lccl} 
Slide & $\theta$ before immersion & Time of immersion & $\theta$ after immersion \\
& \multicolumn{2}{c}{ Stearic Acid Films } \\
A & $44^{\circ} \pm 1^{\circ}$ & 1 min. & $40^{\circ} \pm 1^{\circ}$ \\
B & 44 & 2 & 37 \\
C & 44 & 3 & 37 \\
D & 44 & 5 & 33 \\
H & 44 & 6 & 31 \\
F & 44 & 8 & Not oleoph. \\
& & Arachidic Acid Films & \\
A & $44^{\circ} \pm 1^{\circ}$ & 1 min. & $40^{\circ} \pm 1^{\circ}$ \\
B & 43 & 3 & 39 \\
C & 44 & 3 & 38 \\
D & 44 & 5 & 35 \\
E & 45 & 7 & 32 \\
F & 44 & 8 & Not oleoph.
\end{tabular}


fully compared with patterns from undepleted films. A decrease in the intensity of the layer lines, relative to the background, accompanied the decrease in the contact angles, but there were no detectable changes in the shapes of the layer lines such as would indicate changes in the orientation of the adsorbed molecules.

A few experiments were also carried out with films of monopalmitin and monostearin formed by immersing microscope slides for 5 seconds in $1 \times$ $10^{-3}$ molal solutions of these compounds in the purified cetane. Drops of cetane showed advancing contact angles which averaged $41 \pm 1^{\circ}$ on these films, and electron diffraction patterns of the arced layer line type were obtained from them. The patterns were generally less well defined than the patterns from the acid films and were correspondingly more difficult to interpret. The arcing of the layer lines was found to be somewhat greater than in the patterns of any of the acids, although there was no detectable difference between patterns from the two monoglycerides. The best estimate of the average molecular tilt was $12-15^{\circ}$.

\section{Discussion}

From the contact angle measurements it is concluded that the most important factor in determining the oleophobic properties of films of fatty acids, and similar polar organic compounds, adsorbed on solid surfaces is the density of the adsorbed molecules on the surfaces. Although a quantitative relationship has not been established, it has been shown that the dependence is a direct one such that decreases in the densities of the films are aceompanied by decreases in their oleophobic properties. This is particularly evident from the data of the depletion experiments recorded in Table II. In these experiments, the contact angles exhibited by drops of cetane on films of stearic and arachidic acids decreased from $44^{\circ}$ to nearly $30^{\circ}$ when the films were immersed in pure cetane to dissolve some of the adsorbed molecules from the surfaces. The contact angle variations shown in Table I for films formed from acid solutions of different concentrations are also consistent with this conclusion, for with a constant short period of immersion the number of molecules adsorbed on the surfaces would be expected to increase with the concentrations of the solutions.

It is also concluded that the length of the adsorbed polar molecules is not a major factor in determining the oleophobic properties of the films. The same maximum contact angle of $43-44^{\circ}$ was observed for drops of cetane on films of all the acids which were prepared from their nearly saturated solutions (Table I). Wide variations in the contact angles of drops on films from the same acid were observed in the cases of myristic and lauric acids (Table I) and of arachidic and behenic acids (Table II).

These conclusions are reasonable when it is considered that the oleophobic phenomena must arise from the replacement of the high-energy 
surface of the substrate with a low-energy hydrocarbon surface upon which the hydrocarbon liquids do not spread. Since the van der Waals forces attracting the molecules of liquid to the surface are operative over distances of only a few Angstrom units, it is clearly the characteristics of the outer surfaces of the films and not their thickness which determine their oleophobic properties. For the ideal case of dense, insoluble films on completely smooth substrates, the oleophobic properties should be the same for films of acids only a few carbon atoms in length as for acids of many carbon atoms such as those studied here.

In practice, however, the roughness of the substrate surface may be large compared with the dimensions of the polar molecules, and increasing the length of the molecules may well improve their ability to form films having the required surface characteristics. It is suggested that this accounts for the difference between the present results and those reported previously (1) for films adsorbed on rolled platinum foil which showed an increase in contact angles with increasing chain length of amine films. The glass microscope slides used here were probably smoother than the rolled foil.

The electron diffraction studies show variations in the average tilt of the axes of the polar molecules from the normal to the substrate which are related to the lengths of the molecules. In films of the 14-carbon myristic acid the average tilt is estimated to be $6^{\circ}$ more than in films of the 20-carbon arachidic acid. The molecular tilt also appears to depend on the size of the polar group as indicated by the fact that the tilt of the molecules in films of monostearin and monopalmitin is considerably greater than in films of acids of the same length. The other notable feature observed in the diffraction patterns is that the intensity of the layer lines relative to the background scattering decreases in films treated to give decreasing contact angles.

Consideration of both the diffraction and contact angle observations shows no correlation between the contact angles and the tilt or length of the molecules. Films from the most concentrated solutions of the different acids show the same contact angles but different tilts. Soaking the films in pure cetane reduces the contact angle but does not change the tilt appreciably. The assumption that soaking reduces the surface density of adsorbed molecules is supported by the observed decrease in intensity of the diffraction patterns.

It is of interest to consider the diffraction results in connection with the structural models which have been proposed for the films. The most interesting of these is the micelle model which was proposed by Epstein. This model is generally consistent with the electron diffraction observations and takes into account such general structural features as the randomness in direction of the tilt of the molecules and the occurrence of a range of values 
of tilt. In addition it provides for the increase of the average molecular tilt with a decrease in chain length and with an increase in the size of the polar groups, as was observed here.

The early concept that the outer surfaces of the films must consist of closely packed methyl groups in order for the films to be oleophobic must be modified somewhat. Since the molecules show considerable tilt from the normal to the average surfaces, it is apparent that even in the most dense films considerable numbers of methylene groups of the hydrocarbon chains of the adsorbed molecules must be exposed in the surfaces of the films. Furthermore, the oleophobic properties persist even after considerable depletion of the films when even greater numbers of the methylene groups, and even some areas of the substrate surfaces, are undoubtedly exposed.

It must be emphasized that both of the above models were developed for ideally smooth substrates. Surfaces in practice are nearly always rough on a molecular scale, and this could have a pronounced effect on the proportion of methyl and methylene groups exposed in the surfaces of the films as well as on the energeties of the formation of micelles. The correlation of molecular tilt with chain length should include the influence of surface roughness. The molecular tilt observed in diffraction patterns is measured relative to the average surface and should be used only cautiously in deducing the tilt at the actual point of attachment in deriving structural models. A direct effect of surface roughness on tilt has already been noted (3) for cerotic acid films on platinum in which the observed maximum tilt of $30^{\circ}$ on rough rolled platinum decreased to nearly $0^{\circ}$ when the platinum was given a careful metallographic polish. This effect should be even greater for acids of shorter chain length. Further studies of adsorbed films certainly should include the effect of surface roughness on their structures and oleophobic properties.

\section{ACKNOWLEDGMENT}

The authors wish to express their appreciation to the Procter and Gamble Company for the pure fatty acids used in this research.

\section{REFERENCES}

1. Bigelow, W. C., Pickett, D. L., and Zisman, W. A., J. Colloid Sci. 1, 513 (1946).

2. Bigelow, W. C., Glass, E., and Zisman, W. A., J. Colloid Sci. 2, 563 (1947).

3. Brockway, L. O., and Karle, J., J. Colloid Sci. 2, 277 (1947).

4. Karle, J., and Brockway, L. O., J. Chem. Phys. 15, 213 (1947).

5. Epstein, H. T., J. Phys. \& Colloid Chem. 54, 1053 (1950).

6. Karle, J., J. Chem. Phys. 17, 500 (1949).

7. Williams, R. C., and Epstein, H. T., private communication.

8. MACK, G. L., J. Phys. Chem. 40, 159, 169 (1936).

9. Germer, L. H., and Storks, K. H., J. Chem. Phys. 6, 280 (1938). 